\title{
Aplicación del lenguaje audiovisual en el bachillerato de Industria de la Confección
}

\begin{abstract}
(c) (1) (8)(2)
\end{abstract}
Application of audiovisual language in the bachelor of Clothing Industry

Alexandra Pruna Guachamin. ${ }^{1}$, Fanny Pachacama Chiguano. ${ }^{2} \&$ Mayra Robles Guananga. ${ }^{3}$

Recibido: 20-05-2019 / Revisado: 06-06-2019 /Aceptado: 07-07-2019/ Publicado: 06-09-2019

\begin{abstract}
DOI: https://doi.org/10.33262/cienciadigital.v3i3.3.766

The present investigation is the use of audiovisual media in the training of technical bachelors in the Clothing Industry, the competences and the interest that the educational community gives through the appropriate use of several technological tools at the time of receiving and teaching the module classes technicians at the Quito Fiscal Educational Institution.

The research methodology used is qualitative - descriptive, focused on the application of audiovisual media as pedagogical support in a technical baccalaureate. This information is obtained through interviews with experts and a field newspaper. The comparison of the information obtained gives us the result that the urgent application of technological tools is important to strengthen the knowledge competences considering that it is a very creative baccalaureate and needs to be up to date with this information, this will allow the affirmation of significant learning.

It is understood that the use of these audiovisual media does not guarantee that the student fully learns since the teacher is the one who accompanies the learning, the
\end{abstract}

\footnotetext{
${ }^{1}$ Institución Educativa Fiscal, Quito, alexandra.pruna@educacion.gob.ec

${ }^{2}$ Institución Educativa Fiscal, Quito, fanny.pachacama@educacion.gob.ec

${ }^{3}$ Escuela de Educación General Básica Virginia Larenas, mayra.robles@educacion.gob.ec
} 
student is the only protagonist of creating their own learning with the help of several supports that the teacher applies at the time.

It should also be understood that as a fiscal institution, one cannot have one hundred percent the availability of using equipment and, above all, being able to navigate easily in the educational center.

Keywords: Audiovisual media, technological tools, technical baccalaureate.

\section{Resumen}

La presente investigación es la utilización de medios audiovisuales en la formación de bachilleres técnicos en Industria de la Confección, las competencias y el interés que da la comunidad educativa por medio del uso adecuado de varias herramientas tecnológicas al momento de recibir e impartir las clases de módulos técnicos en la Institución Educativa Fiscal Quito.

La metodología de investigación utilizada es cualitativa - descriptiva, enfocado a la aplicación de los medios audiovisuales como soporte pedagógico en un bachillerato técnico. Dicha información se la obtiene por medio de las entrevistas a expertos y un diario de campo. El cotejo de la información obtenida nos da como resultado que es importante la aplicación urgente de herramientas tecnológicas para fortalecer las competencias del conocimiento considerando que es un bachillerato muy creativo y necesita estar al día con dicha información esto permitirá la afirmación de un aprendizaje significativo.

Se entiende que el uso de estos medios audiovisuales no garantiza que el estudiante aprenda completamente ya que el docente es quien acompaña el aprendizaje, el estudiante es el único protagonista de crear su propio aprendizaje con la ayuda de varios soportes que el maestro aplique en su momento.

También hay que entender que por ser una institución fiscal no se podrá tener al cien por ciento la disponibilidad de utilizar equipos y sobre todo de poder navegar de forma fácil en el centro educativo.

Palabras Claves: Medios audiovisuales, herramientas tecnológicas, bachillerato técnico. 


\section{Introducción}

Realizando un diagnostico profundo sobre el uso de las herramientas tecnológicas en el bachillerato técnico en Industria de la Confección se aprecia el problema que muy pocos docentes incorporan algunos entornos virtuales para el aprendizaje como: preparación de evaluaciones, recursos o materiales didácticos, simuladores, entre otros, es posible que ciertos maestros no apliquen este aprendizaje como una nueva opción de enseñanza por falta de conocimiento o por no contar con los medios tecnológicos en la institución, a su vez se menciona también que en este bachillerato existen muy pocas plataformas de uso práctico, es por ello de esta investigación, en el cual se pretende cambiar la mentalidad de ciertos docentes que desconocen varias aplicaciones prácticas para el desarrollo de los módulos de trabajo, motivando el transcurso de aprendizaje de todos los estudiantes quienes aplican por esta opción educativa.

Se considera que al seguir trabajando de forma tradicional no se estará a la vanguardia de la sociedad tecnológica ni de los estudiantes que cada vez van incursionando a mejorar su conocimiento y más si se trata de una herramienta muy interactiva entre los alumnos de cualquier parte del mundo quienes comparten el mismo gusto.

Se cree que al trabajar con algunos de los entornos virtuales cambiará el aprendizaje tradicional de cada uno de los estudiantes fortaleciendo su competencia del saber - hacer, se busca implementar a través de herramientas tecnológicas audiovisuales, diseñadas en este caso específicamente para el área educativa mediante una página web llamada JIMDO, con el fin de optimizar la formación de bachilleres técnicos en la figura profesional Industria de la Confección, a través de un proceso tecnológico e interactivo, facilitando en este sentido una filosofía de aprendizaje real a través del lenguaje audiovisual, en donde los estudiantes sean los promotores de su deseo de formarse, buscar, leer e innovar cada día con el uso de una herramienta digital que se adecue a las necesidades globales de los mismos.

Cabe destacar que la importancia científica de esta investigación mediante un proceso de revisión exhaustiva de texto escrito por especialistas en el área pedagógica, didáctica y de los diferentes puntos de vista de cómo es el proceso para que se logre un aprendizaje, se desarrolla 
una herramienta digital interactiva con lineamientos pedagógicos y didácticos específicos para el área donde se observa el problema como fue el del bachillerado técnico en la Industria de la Confección.

Con esta herramienta digital se pretende que los estudiantes se interesen y se apropien de esta nueva metodología de enseñanza demostrando un mejor uso de la tecnología para su aprendizaje profesional, personal, cultual, social y económico considerando que en el país hace falta gente conocedora de estas nuevas aplicaciones informáticas con esto se propone que los bachilleres técnicos sean los pioneros en toda investigación y aplicación de prendas en las cuales sean los creadores de nuevas tendencias de moda.

Considerando las necesidades de los estudiantes se plantea una interrogante, ¿cómo mejorar el aprendizaje de un estudiante técnico?, la respuesta puede dar origen a varias investigaciones, abarcaría entonces en este análisis realizar algunos cuestionamientos sobre el posicionamiento de un lenguaje virtual que sea aporte de mejora en este proceso de quienes cursan este bachillerato como: ¿Es creatividad el uso de un medio tecnológico?; ¿Qué características debe tener una plataforma de diseño para un bachiller técnico?; ¿Es pedagógico utilizar un medio audiovisual?

\section{Desarrollo}

El gran mundo de la moda obliga a estar pendientes de las últimas e innovadoras tendencias y tecnologías en cortes, líneas, texturas, colores, tejidos y accesorios con el único objeto de cambiar la presentación de la figura humana. Thomas Carlyle dice: "El hombre está compuesto por cuerpo, alma y vestido", lo que convierte a esta profesión en una necesidad primordial del ser humano. Se puede decir que la moda es un ente que tiene vida porque todo el tiempo está en movimiento, nunca se detiene y en cada momento hay algo nuevo por hacer, permitiendo a las personas desarrollar potencialidades.

Otro factor importante dentro de este campo profesional es la posibilidad real con la que cuentan las futuras profesionales para poder tener su propio negocio o empresa; así lo reconocen también grandes personalidades de la moda como lo es Yolanda Voss, (una gran diseñadora ecuatoriana que trabaja en los estados unidos, quien manifiesta que "El diseñador es un artista 
que debe conocer de mercadeo, de promoción, tecnología y otras tantas cosas más que a la larga aprendemos y es como vender nuestra propia creatividad".

Según (Vejar, 2014) menciona que: “...los cambios sucedidos en la constante dinámica de acumulación del capital muestran sorprendentes efectos a la hora de evaluar los impactos en el trabajo y el empleo". Los bachilleres técnicos deben estar preparados para toda actualización que a diario se vive en un mundo en donde la tecnología es tan variante, solo así se podrá estar a la altura de las competencias laborales que exige el mundo de la moda.

Por lo tanto, las clases deben estar encaminadas a formar un pensamiento y un aprendizaje reflexivo que se convierten en procesos de investigación interactiva. (Iglesias, 2010) Según lo expuesto en el presente proyecto ha de contribuir a un "aprendizaje interactivo" que se propone conseguir la propuesta de renovar el ciclo de aprendizaje en el bachillerato técnico en Industria de la Confección a través de la aplicación de una estrategia mediada por las TIC para lograr una excelente preparación de los estudiantes técnicos dentro de una educación y formación que aporte a la matriz productiva nacional.

Para (del Moral Pérez, 1999) menciona la importancia de escoger una herramienta digital acorde al trabajo a desempeñar. "La selección del software que debe utilizarse con los estudiantes es vital. Es necesario decidir qué tipo de programas contribuirán a desarrollar la capacidad creativa de los alumnos."

Al hablar de un fundamento pedagógico se piensa en una organización de varios procesos que van direccionan a un mismo lugar con un mismo fin del como saldrá el estudiante que siga industria de la confección el mismo que se siguen las competencias propias, según el año que vayan cursando. Se contempla en esta investigación un proceso metodológico de tres fases: que cumpla la competencia en cada uno de los módulos de estudio, reestructuración de los módulos de trabajo, validación de la reestructuración de los módulos con aplicación de las TIC. Todos estos momentos basados en las competencias establecidas en el ministerio de educación las cuales corresponderán a la elaboración de planificaciones y programas utilizando con una visión de producción y funcionamiento en cada una de las aulas siendo actualizadas en base a proyectos didácticos productivos. 
Las clases deben estar encaminadas a formar un pensamiento y un aprendizaje reflexivo que se convierten en procesos de investigación interactiva. (Ruiz Iglesias, 2010, pág. 112.) Según lo expuesto el presente proyecto ha de contribuir a un "aprendizaje interactivo" que se proyecta a obtener una mejora en el proceso de enseñanza aprendizaje en el Bachillerato Técnico en Industria de la confección a través de la aplicación de una estrategia mediada por las TIC para lograr una excelente preparación de los estudiantes técnicos dentro de una educación y formación que aporte a la matriz productiva nacional.

\section{Los Medios Audiovisuales}

Según (Tomás, 2009) dice que: “...los medios audiovisuales son instrumentos tecnológicos que ayudan a presentar información mediante sistemas acústicos, ópticos o una mezcla de ambos. Es importante reconocer que los jóvenes en la actualidad nacen con la tecnología y les es más fácil integrar y utilizar ciertos medios audiovisuales destacando que no es tan sencillo el interpretar una imagen o un sonido para ello están los docentes quienes son los que acompañan en el proceso de enseñanza aprendizaje. El uso permite que el estudiante absorba toda la información posible utilizando sus sentidos como la vista y el oído".

\section{Lenguaje Audiovisual como herramienta didáctica}

Según la historia se dice que los medios audiovisuales llegaron a cambiar la sociedad humana conforme fueron apareciendo durante el siglo XX, en la actualidad son el origen principal de toda información dada por soportes digitales como las páginas Web, eso hace que la llegada del internet cambie todo pensamiento limitante de estos lenguajes los mismos que se basan es estimular los estímulos que buscan imitar la realidad o realizar una nueva ficción.

(Bartolomé, 1987) Menciona que: “Al lenguaje audiovisual se lo puede entender cómo, las músicas, efectos sonoros, los ruidos y los silencios colaboran en la transmisión del mensaje, no como fondo o complemento sino conformando realmente el mensaje". Cada uno llega a ser parte del individuo en donde el conocimiento se fortifique en esta sociedad tan cambiante en el cual es un aprendizaje más actual al momento de interactuar de forma inmediata y en cualquier parte del mundo. 


\section{TIC en el Sistema Educativo Ecuatoriano}

Si se habla de TIC no se puede dejar de lado el pensar en una nueva forma de vida, en la década de los 80 aparece el uso de computadores el cual llevo a que se vaya utilizando el internet, redes sociales hasta llegar a la inteligencia artificial. (Castellanos-Niño, 2012; Garcés-Pretel \& RuizCantillo, 2016).

\section{Recursos tecnológicos Tic para el lenguaje audiovisual}

Los recursos tecnológicos son actualmente una tendencia que entrega numerosas formas para poder aprender a la hora de dar clases, dando la posibilidad de adquirir nuevas competencias, ampliando a una sociedad interactiva, dejando de lado los medios tradicionales. Conociendo que la educación en materia de comunicación se aplica hasta los años noventa y lo audiovisual para fines educativos a partir del siglo XXI.

La tecnología se utiliza como herramienta para favorecer las competencias, en todos los niveles educativos, es decir desde parvulario hasta la universidad, donde el uso del pizarrón es un claro ejemplo para mostrar la manera de resolver un problema sencillo o complejo, con el advenimiento de la tecnología, esas herramientas se han diversificado, dando paso a un proceso de aprendizaje en donde se realizan varias propuestas de que es mostrar y como realizarlo, sinterizándose en el reconocimiento de que mostrar es visualizar y realizar para que se pueda entender, es decir se cumple con la competencia de aprender haciendo, tal cual lo requiere el bachillerato Industria de la confección.

Tabla 1. Comparación entre Páginas Web

\begin{tabular}{|c|c|c|c|}
\hline N. & Herramienta & $\underline{\text { Costo }}$ & Prestaciones \\
\hline \multirow[t]{4}{*}{1} & Jimdo & Gratuito (Básico) & Su uso es sencillo \\
\hline & & Para mejorar el servicio requiere pago adicional & Se adapta a los móviles \\
\hline & & & Sus plantillas son muy rígidas \\
\hline & & & Tienda online \\
\hline \multirow[t]{4}{*}{2} & Weebly & Gratuito (Básico) & Facilidad de uso \\
\hline & & Para mejorar el servicio requiere pago adicional & Plantillas adaptables \\
\hline & & & $\begin{array}{l}\text { Gestión de equipo } \\
\text { No tiene Diseños inflexibles }\end{array}$ \\
\hline & & & No tiene Páginas multilingües \\
\hline
\end{tabular}


3

Squarespace

No es gratuito
Plantillas y diseño con Soporte

Es excelente para crear blogs

Su versión no está en español

El costo es alto

No tiene vista previa

Nota: Análisis de información para selección y utilización de una página que sea de uso y aporte para estrategias de aprendizaje en un bachillerato técnico de figura profesional industria de la confección.

Como medios audiovisuales utilizados en esta investigación se comparan Jimdo, Weebly y Squarespace los cuales han sido analizados como estrategia pedagogía para ser aplicados en el aula de clases, fortaleciendo las competencias y valores en el bachillerato técnico en industria de la confección los cuales optimizaran el uso de las TIC en la formación de cada estudiante en su diario vivir, en su parte académica y profesional a lo cual se escoge Jimdo por ser una página gratuita y de fácil utilidad.

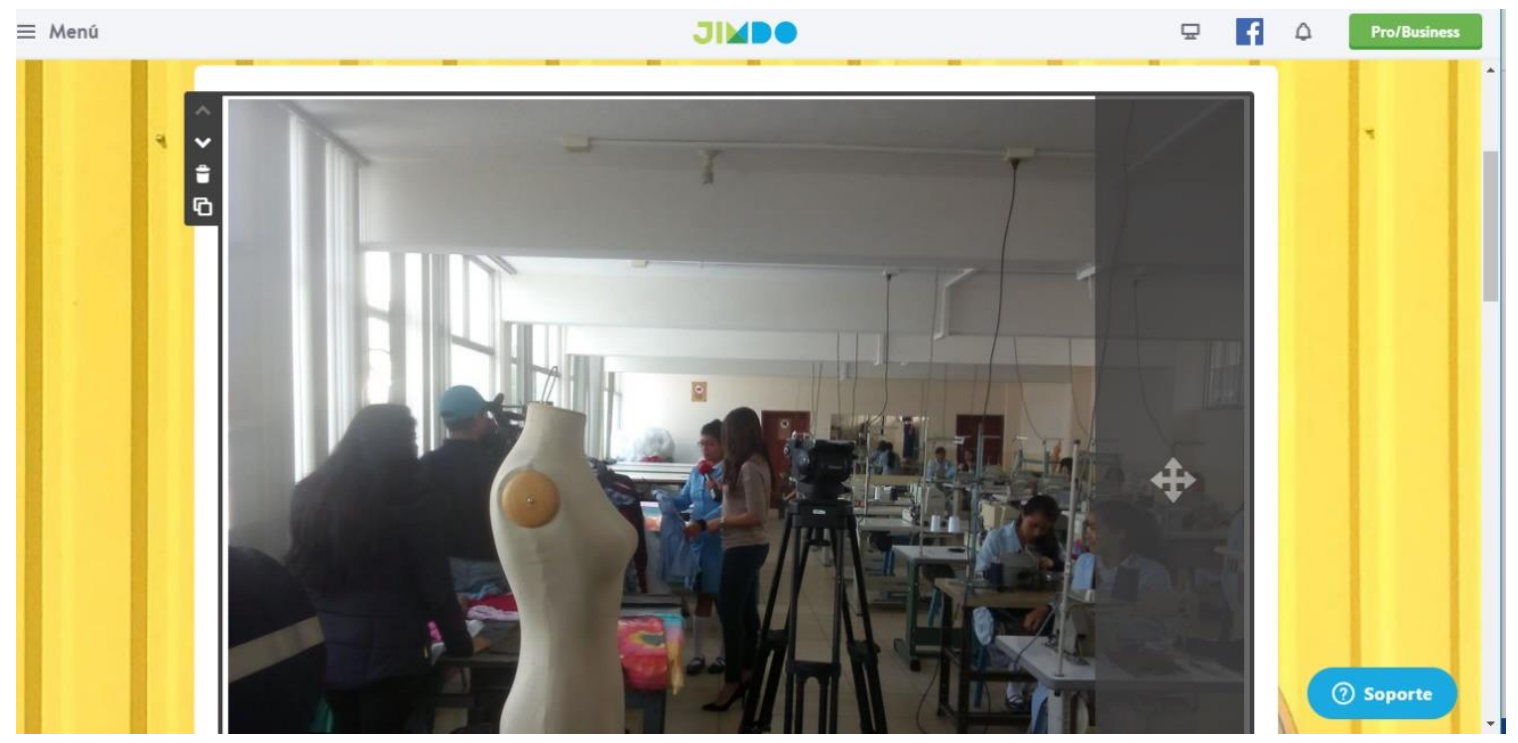

Figura 1. Fotografía Inicial de la Pagina Jimdo

Los sitios web de recursos y datos educativos también son de naturaleza informativa que va proporcionando a los usuarios fundamentos en forma de enlaces, documentos, direcciones, recursos, software, entre otros. En la actualidad existe una gran variedad de sitios web como son las hemerotecas virtuales de revistas educativas, base de datos de investigaciones, de publicaciones de documentos, web personal de docentes, o portales de información educativa como: educaweb, eduteca, maestroteca todos estos son páginas web informativos. 
En su gran mayoría las plataformas virtuales han sido diseñadas para la organización de recursos académicos, considerando que tienen varias actividades como foros y chats, los que proporcionan que se den entornos interactivos con varios estudiantes tomando el control de su propio aprendizaje y así puedan crear su criterio determinando y sus conclusiones de aprendizaje.

Jimdo aparece en el 2007 y es de origen alemán, en esta plataforma se puede crear páginas web de manera sencilla el cual está basado en unidades editables, cuenta con su propio sistema de gestión de contenido. Esta plataforma es más visual y no requiere un editor de páginas web como HTML, aunque si lo permite por lo tanto no es importante tener conocimiento de creación de páginas web, aunque se puede realizar algunas modificaciones de diseño en cada una de las plantillas o hacerlo más personalizado.

Dispone de tres versiones:

- JimdoPro

- JimdoBusiness

- JimdoFree

Tabla 2. Página Web Jimdo

\section{Ventajas}

- Fácil de utilizar, se requiere poco o nada de conocimiento en diseño web. Es sencillo

- Cuenta con un foro interno el cual ayuda a encontrar soluciones al momento de crear o mantener una página web.

- Tiene un servicio de atención al cliente

- Jimdo ofrece el nombre de dominio en dos formas de pago

- Las plantillas de Jimdo se pueden visualizar desde cualquier dispositivo

\section{Desventajas}

- Sus plantillas son poco personalizables

- La versión gratuita contiene mucha publicidad

- No se puede crear copias de seguridad de diseño o contenidos

- Jimdo no tiene aplicaciones extra

- La velocidad del soporte técnico no es la correcta

Nota: aspectos importantes que se debe considerar antes de utilizar esta herramienta 


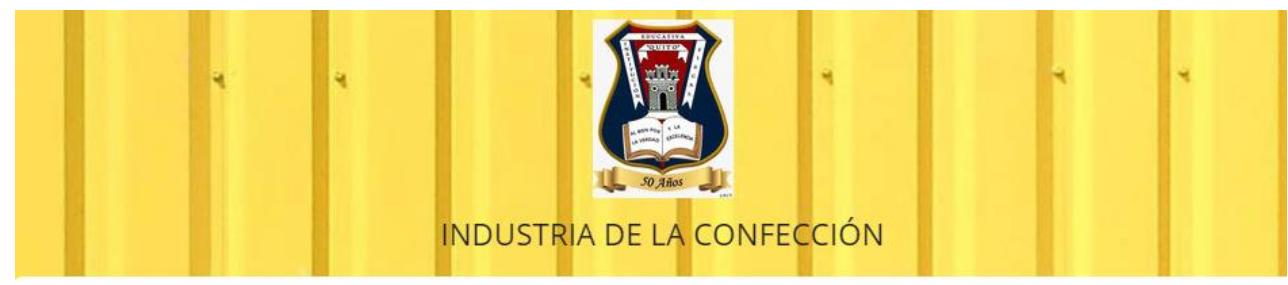

\section{Qué es el Patronaje}

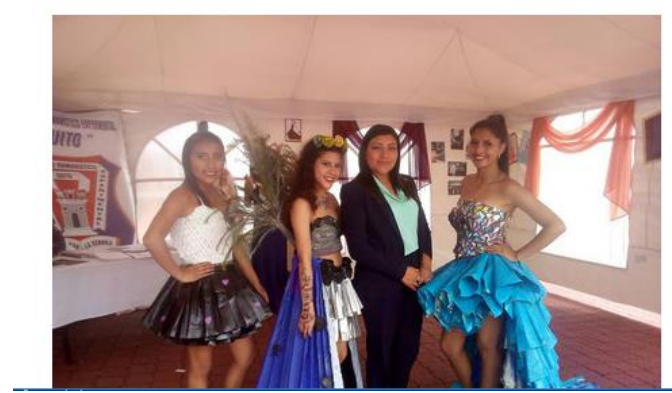

Se llama patronaje a la actividad de diseñar y adaptar patrones.

Patronista es el profesional que se dedica al patronaje. Esta profesión surge con la industrialización del proceso de confección. Anteriormente, la persona que los realizaba era el sastre o la modista que trazaba el patrón a medida, para una persona determinada en lugar de usar tallaje comercial.

Figura 2. Aplicación de Temas Relacionados con los Módulos de Trabajo en Jimdo

\section{Métodos}

(LÓPEZ NOGUERO, 2002) Menciona que "para realizar un trabajo de investigación es necesario llevar a cabo un desarrollo metódico que permita la evacuada consecución de los objetivos propuestos, así como una formulación clara, concreta y precisa del problema y una metodología de investigación rigurosa y adecuada al tipo de trabajo" una vez identificado el problema y determinado lo que se pretende con este estudio se opta como metodología la más adecuada para poder llegar a cumplir con éxito los objetivos planteados.

El método que se utiliza en este estudio es cualitativo, el mismo que se define como:

"Cualquier tipo de investigación que produce resultados a los que no se ha llegado por procedimientos estadísticos u otro tipo de cuantificación. Puede referirse a investigaciones acerca de la vida de las personas, historias, comportamientos, y también al funcionamiento organizativo, movimientos sociales o relaciones e interacciones. Algunos de los datos pueden ser cuantificados pero el análisis en sí mismo es cualitativo" (STRAUSS y CORBIN, 2002), por otra parte (Aliaño, 2014) asegura que es un estudio donde el análisis se realiza como aceptar un contexto, sin esperar los resultados no determina una investigación. Es por eso que en la presente investigación el análisis se realiza al uso de medios audio visuales como soporte de las nuevas tecnologías del bachillerato técnico industrial con estudiantes de primero a tercero del 
bachillerato en Industria de la Confección ubicados en la provincia de Pichincha, refiriendo los aspectos más relevantes de esta necesidad.

Se pone en consideración los resultados a través de la comparación de los datos obtenidos de las entrevistas, esto llevara a realizar una propuesta de aplicación de medios audiovisuales en un bachillerato técnico se estudia la intervención de estas herramientas tecnológicas comprendiendo el valor científico de los resultados obtenidos.

La importancia de esta investigación es motivar al estudiante a prepararse e interesarse sobre los módulos de trabajo de dicho bachillerato con una metodología que no solamente son valores numéricos ni generalizables sobre el uso de medios audiovisuales y TIC, sino que indique la utilidad de emplear estos medios mejorando el aprendizaje en los estudiantes.

Este tipo de estudio cualitativo se lo realiza tomando en cuenta el tipo de trabajo realizado en las aulas en una clase tradicional en donde se limita el impartir las clases directamente con el uso de la pizarra, el método de enseñanza es basado en los proyectos (ABP), esto permite al estudiante que den respuesta a los problemas de la vida real, garantizando los procesos de aprendizaje más didácticos, apoyados en esta aplicación será de gran apoyo investigativo y de ayuda para entender la importancia científica de los resultados obtenidos.

Las derivaciones de los resultados que se obtienen por medio del cotejo de datos obtenidos a través de la entrevista y un diario de campo, esto será parte para poder realizar una nueva propuesta de inclusión basada en el uso de medios audiovisuales analizando la propuesta del comportamiento de los estudiantes quienes serán los que utilicen en la hora de clases.

\section{Instrumentos de investigación}

Se indica el estudio a través de la entrevista

Según (RUÍZ, 2012) la entrevista nace a partir del desconocimiento del entrevistador el cual desea conocer los actos de los sujetos, esto permite que puedan explicar con profundidad cada una de sus respuestas, a lo cual el autor sigue manifestando sobre la entrevista que:

Por su propia naturaleza, introduce un elemento nuevo al colocar un intermediario entre el fenómeno y el investigador. El fenómeno examinado en una entrevista es conocido a través del relato (descripción, interpretación, explicación) el entrevistado que somete 
la realidad a un proceso de reelaboración, ente lo que ocurre y el observador que lo quiere estudiar (p.127)

Este tipo de técnica es la que se utiliza la investigación considerando que en la propuesta planteada no se puede encontrar mayor información y se pretende cambiar la manera de llegar al estudiante a motivarlo y por supuesto que como docentes puedan aplicar otra forma de dictar sus clases.

La entrevista se lo realiza a tres personas quienes están de forma directa trabajando en el bachillerato técnico. El tipo de entrevista que se ejecuta es la conversación en profundidad, misma que se identifica por ser de estructura abierta, holística e individual. Se realiza las tres entrevistas en forma individual al analista de currículo de educación técnica del Ministerio de Educación, a la Coordinadora del Área Técnica del colegio Gran Colombia y a la Vicerrectora del colegio Nicolás Jiménez. El carácter holístico se refiere a los puntos de vista en múltiples interacciones que se analizaran de forma general y no de forma individual según el criterio del entrevistado, por otro lado es abierta a pesar de que las preguntas estén planteadas de esta manera se espera que el entrevistado pueda responder confiadamente y se llegue a obtener la información necesaria para sustentar nuestro estudio en cual se pueda corroborar la necesidad de utilizar los medios audiovisuales en un bachillerato técnico.

Las entrevistas se hacen a personas que están desde el campo de acción en donde trabajan directamente con los estudiantes y las aulas, este conversatorio será evidenciar la necesidad que como docentes están expuestos en el momento de dictar las clases el poder llegar a los educandos de una forma atractiva y que sus conocimientos sean consolidados efectivamente en cada uno de los estudiantes a la vez demostrando que la tecnología es imprescindible en nuestra actualidad.

Para llegar a utilizar la entrevista se toma como guía a lo que según menciona (Álvarez -Gayou, 2005) realizando preguntas claras relacionadas entre sí.

\section{Diario de campo}

Según (Spradley, 1980) dice que el diario de campo "contendrá un registro de experiencias, idea, miedos, errores, confusiones, soluciones, que surjan durante el trabajo de campo. El diario constituye la cara personal de ese trabajo, incluye las reacciones hacia los informantes, así como los afectos que uno siente que le profesan los otros" 
Por medio de este diario de campo se pretende obtener toda la información detallada y ordenada sobre la utilización de dicha metodología antes y después del uso de los medios audiovisuales, del docente, los estudiantes las cosas que se desarrollan en el lugar de clases (el ambiente, formas de pensar, desarrollo y forma de enfrentar los problemas). Cada una de las observaciones son escritas y apuntadas en el diario de campo, en este se va a describir cada una de las situaciones ya sean personales o grupales esto es importante ya que se pretende reflexionar sobre el uso de la tecnología en la práctica educativa y poder obtener el resultado de dicha investigación.

\section{Resultados}

De acuerdo a los resultados obtenidos se puede detectar lo siguiente:

Preguntas realizadas en la entrevista

1. ¿Qué le parece la incorporación de las nuevas tecnologías en la educación técnica?

2. Conoce alguna página web o portal que sirva de aporte en este bachillerato

3. Según los criterios de evaluación del bachillerato técnico se podría utilizar algún entorno virtual para su pronta aplicación y retroalimentación.

4. Recomendaría usted la aplicación de herramientas tecnológicas en este bachillerato

5. Conocedores de un bachillerato que maneja competencias, ¿cree que es importante realizar las clases de forma más interactiva y utilizar varios entornos virtuales?

Tabla 3. Matriz de evaluación de resultados de encuesta

\begin{tabular}{|c|c|c|c|c|c|}
\hline & $\underline{\text { Pregunta } 1}$ & Pregunta 2 & Pregunta 3 & Pregunta 4 & Pregunta 5 \\
\hline Entrevistado 1 & $\begin{array}{l}\text { Importante } y \\
\text { necesario para } \\
\text { contribuir a un } \\
\text { proceso de } \\
\text { formación no } \\
\text { solo local sino } \\
\text { fuera }\end{array}$ & $\begin{array}{l}\text { Vinculado a } \\
\text { modas no. } \\
\text { La vinculación no } \\
\text { es tan cercana }\end{array}$ & $\begin{array}{l}\text { Es muy buena } \\
\text { la propuesta }\end{array}$ & $\begin{array}{l}\text { Claro que si ya } \\
\text { que en el diseño } \\
\text { de modas es } \\
\text { muy creativo }\end{array}$ & $\begin{array}{l}\text { De gran manera } \\
\text { si ya que es } \\
\text { innovador el uso } \\
\text { de herramientas } \\
\text { en una práctica } \\
\text { de clases }\end{array}$ \\
\hline Entrevistado 2 & Muy innovador & Blogs de modas & $\begin{array}{l}\text { Sería lo más } \\
\text { optimo }\end{array}$ & $\begin{array}{l}\text { Por supuesto } \\
\text { que si la moda } \\
\text { va de la mano } \\
\text { con la } \\
\text { tecnología }\end{array}$ & $\begin{array}{l}\text { Podrían } \\
\text { vivenciar la } \\
\text { realidad en la } \\
\text { práctica laboral. }\end{array}$ \\
\hline Entrevistado 3 & $\begin{array}{l}\text { Importantes } \\
\text { necesarias }\end{array}$ & $\begin{array}{l}\text { No en medida de } \\
\text { formación } \\
\text { académica }\end{array}$ & $\begin{array}{l}\text { De existir una } \\
\text { propuesta } \\
\text { sería muy útil }\end{array}$ & $\begin{array}{ll}\text { Desde luego } \\
\text { que si }\end{array}$ & $\begin{array}{l}\text { Es } \\
\text { imprescindible } \\
\text { si se desea estar }\end{array}$ \\
\hline
\end{tabular}


Vol. 3, N³.3, p. 01-18, septiembre, 2019

\begin{tabular}{|c|c|c|c|c|c|}
\hline & & & & & $\begin{array}{l}\text { acordé a un } \\
\text { mundo } \\
\text { globalizado y } \\
\text { tecnificado }\end{array}$ \\
\hline RESULTADOS & $\begin{array}{l}\text { Se demuestra } \\
\text { que la } \\
\text { innovación } \\
\text { tecnológica es } \\
\text { importante en } \\
\text { el área técnica }\end{array}$ & $\begin{array}{l}\text { Lamentablemente } \\
\text { se conoce muy } \\
\text { poco en medios } \\
\text { audiovisuales }\end{array}$ & $\begin{array}{l}\text { La propuesta } \\
\text { es agradable a } \\
\text { pesar de los } \\
\text { costos de } \\
\text { utilidad }\end{array}$ & $\begin{array}{l}\text { Los expertos } \\
\text { coinciden con } \\
\text { la aplicación de } \\
\text { estas } \\
\text { herramientas }\end{array}$ & $\begin{array}{l}\text { Para estar a la } \\
\text { vanguardia de la } \\
\text { educación es } \\
\text { muy necesario } \\
\text { que se puedan } \\
\text { aplicar } \\
\text { garantizar que } \\
\text { las } \\
\text { competencias } \\
\text { sean aprendidas } \\
\text { de forma } \\
\text { óptima. }\end{array}$ \\
\hline
\end{tabular}

Nota: cuadro comparativo de respuestas de los entrevistados para su respectivo análisis

El analista de educación técnica comprometido con su labor de mejorar cada vez las competencias no solo de los estudiantes sino del gestor del aprendizaje ya que si un docente es preparado previamente puede desempeñarse en el aula de clases y aplicar actividades que genere el interés por aprender. Manifiesta que sobre el uso de los medios audiovisuales y TIC en el área técnica ha sido muy escasa que desde su posición como organizador y realizador de currículo no hay mayor información, además añade que le parece muy importante que se trate de incluir la tecnología en este bachillerato necesita para mejorar y ser conocidos en un entorno emprendedor y participativo en la esta sociedad actual.

Como coordinadora del Área Técnica en Industria de la Confección cree que el aporte del Ministerio de Educación sería muy importante ya que es imprescindible este tipo de herramientas a la hora de impartir las clases el aporte serio grande ya que los estudiantes son nativos digitales esto haría que se esté a la vanguardia en una sociedad tecnológica.

La tercera entrevistada como autoridad y docente técnica cree que la aplicación de los medios audiovisuales como estrategia de aprendizaje aplicada en el currículo fortalecerá los conocimientos de los estudiantes técnicos independientemente de los módulos de estudio considerando que no todo se llega a la práctica y con la ayuda de estos medios sería de gran aporte para las competencias de aprendizaje. 


\section{Discusión}

Luego de haber realizado el cotejo de resultados de la entrevista se entiende que es de vital importancia la aplicación de medios audiovisuales en un bachillerato tan prometedor e inclusivo en la sociedad, la propuesta de investigación es otorgar herramientas útiles en el aula, evitar caer en la monotonía de toda clase tradicional sino de motivar al estudiante a que su aprendizaje sea completo y sea él quien investigue para que pueda construir su propio conocimiento.

Como docente técnico se corrobora que la educación ha puesto limitante en poder dar un aprendizaje más actualizado o vincular herramientas tecnológicas, lamentablemente el ministerio de educación no abarca completamente las necesidades educativas, es por ello de la propuesta de buscar e implementar una forma distinta de llegar al estudiante de una manera más interactiva, sobre todo que las competencias desarrolladas en el este proceso se queden interiorizado para que luego pueda ser aplicado.

\section{Conclusiones}

Una vez finalizada la investigación se ha llegado a la conclusión que, es importante y necesario que toda planificación, se realice tomado en cuenta la aplicación de recursos tecnológicos, utilizando diferentes métodos didácticos para el aprendizaje, preponderando técnicas de aprender haciendo como competencia indispensable, las cuales deben estar enmarcadas en la necesidad propia del estudiante, para que él como protagonista sea quien obtenga conocimientos a través de medios audiovisuales mejorando su proceso de enseñanza y el docente como mediador sea quien facilite estas herramientas y los medios audiovisuales a la hora de las clases marcando la diferencia en el desarrollo de las competencias técnicas.

Se entiende que las herramientas tecnológicas no siempre serán una innovación en educación, sino que serán de aporte en el momento de solidificar conocimientos y motivar al estudiante a que genere su propio aprendizaje enmarcados en la correcta utilización de estos medios.

El uso como herramienta en el aula no garantiza que como estudiante aumente su motivación o su rendimiento escolar cada una de las herramientas propuestas en esta investigación es adaptada a un módulo de aprendizaje que colabore al aprendizaje y a la vez que como herramienta ayude al docente a llegar a cumplir con el propósito de la clase. 
Vol. 3, N³.3, p. 01-18, septiembre, 2019

Un magnifico aprendizaje es aplicar una estrategia o método de enseñanza en cada una de las planificaciones didácticas. El método de proyectos y el uso de herramientas tecnológicas harán que faciliten el aprendizaje por medio del descubrimiento convirtiéndose en constructivista tal como indica la competencia de este bachillerato del saber hacer.

La competencia del bachillerato tu bachillerato exige que se aprenda haciendo, ahora se debe incluir a los medios audiovisuales mediante el desarrollo de tareas como el buscar y crear información, tener evaluaciones inmediatas, resolver cuestionarios, observar videos, debates virtuales, foros entre otros.

Es importante que como docente técnico estar a la vanguardia de la educación y poder contribuir con los estudiantes haciéndolos competentes en un área tan creativa como el diseño de modas que va de la mano con la tecnología esto hará que sean capaces de tener un criterio de análisis y toda información que pueda seleccionar sea de utilidad para su carrera profesional, las TIC son un recurso de apoyo.

\section{Referencias}

Aliaño, M. \&. (2014). Integración de medios audiovisuales y TIC en educación infantil: estudio de un caso en el Centro Infantil". Andalucia.

Álvarez -Gayou, J. L. (2005). “Cómo hacer investigación cualitativa. Fundamentos y metodología”. Paidos, Mexico .

Bartolomé, A. R. (1987). Lenguaje audiovisual-Mundo audiovisual. . Obtenido de Lenguaje audiovisual-Mundo audiovisual. : http://www.quadernsdigitals.net/datos/hemeroteca/r_48/nr_508/a_6905/6905.pdf

Buitrago, A. N. (2015). La educación médiatica y los profesionales de la comunicacion . Barcelona, España: Gedisa S. A.

del Moral Pérez, M. (1999). Tecnologías de la Información y la Comunicación (TIC). Creatividad y Educación. Educar, 52.

Iglesias, M. R. (2010). El concepto de competencias desde la complejidad: hacia la construcción de competencias educativas. En M. Iglesias, El concepto de competencias desde la complejidad: hacia la construcción de competencias educativas (pág. 112). Trillas.

Litwin, E. (2009). Tecnologías Educativas en tiempo de internet. Buenos Aires, Argentina: Amarrortu. 
LÓPEZ NOGUERO, F. (2002). El análisis de contenido como método de investigación. Revista de educación 4, 167-179.

RUÍZ, J. I. (2012). “Metodología de la investigación cualitativa”. Bilbao: Universidad de Deusto.

Spradley, J. (1980). “Participant Observation”. New York: Holt, Rinehart and Winston.

STRAUSS y CORBIN, J. (2002). "Bases de la investigación cualitativa. Técnicas y para desarrollar la teoría fundamentada”. Medellin, Colombia: Universidad de Antoquia.

Tomás, A. A. (2009). Medios Audiovisuale en el Aula. Pedagogia de los medios audiovisuales. 19.

Vejar, D. (2014). La precariedad laboral, modernidad y modernización capitalista. Trabajo y Sociedad, (23), 147-168.

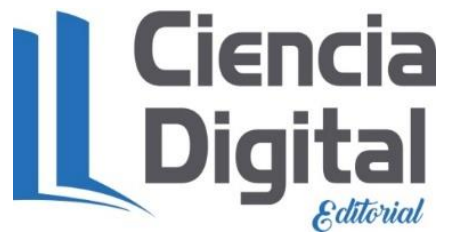




\section{PARA CITAR EL ARTÍCULO INDEXADO.}

Pruna Guachamin, A., Pachacama Chiguano, F., \& Robles Guananga, M. (2019). Aplicación del lenguaje audiovisual en el bachillerato de Industria de la Confección. Ciencia Digital, 3(3.3), 1-18. https://doi.org/10.33262/cienciadigital.v3i3.3.766

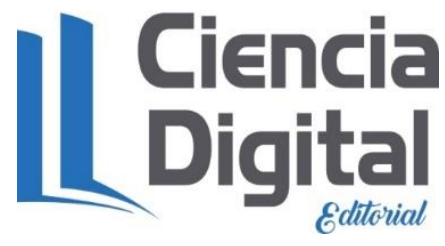

El artículo que se publica es de exclusiva responsabilidad de los autores y no necesariamente reflejan el pensamiento de la Revista Ciencia Digital.

El artículo queda en propiedad de la revista y, por tanto, su publicación parcial y/o total en otro medio tiene que ser autorizado por el director de la Revista Ciencia Digital.
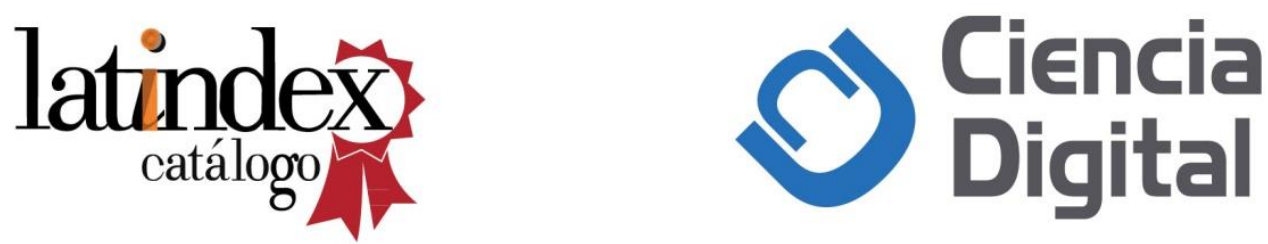